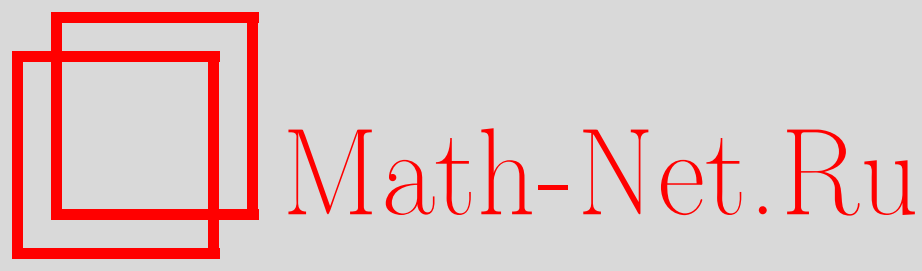

В. А. Москаленко, П. Ентель, Л. А. Дохотару, Р. Читро, Диаграммная теория для примесной модели Андерсона. Свойство стационарности термодинамического потенциала, ТМФ, 2009, том 159, номер 1, 162-173

DOI: https://doi.org/10.4213/tmf6339

Использование Общероссийского математического портала Math-Net.Ru подразумевает, что вы прочитали и согласны с пользовательским соглашением http: //www . mathnet.ru/rus/agreement

Параметры загрузки:

IP : 54.197 .130 .99

26 апреля 2023 г., 14:10:14

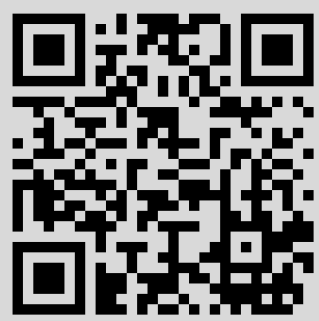




\title{
ФИЗИКА
}

Том 159, № 1

апрель, 2009

\section{9 г.}

В. А. Москаленко*†, П. Ентель ${ }^{\ddagger}$, Л. А. Дохотару ${ }^{\S}$, Р. Читро

\section{ДИАГРАММНАЯ ТЕОРИЯ ДЛЯ ПРИМЕСНОЙ МОДЕЛИ АНДЕРСОНА. СВОЙСТВО СТАЦИОНАРНОСТИ ТЕРМОДИНАМИЧЕСКОГО ПОТЕНЦИАЛА}

\begin{abstract}
Предложена диаграммная теория в окрестности атомного предела для нормального состояния примесной модели Андерсона. Новый диаграммный метод опирается на теорему Вика для электронов проводимости и на обобщенную теорему Вика для сильно коррелированных электронов примеси, которая совпадает с определением кумулянтов Кубо. Доказана теорема о связных диаграммах для среднего значения оператора эволюции, и для одночастичных пропагаторов электронов получены уравнения типа уравнения Дайсона. Основным элементом этих уравнений является корреляционная функция примесных электронов, которая содержит спиновые, зарядовые и парные флуктуации системы. Термодинамический потенциал системы выражен через полный пропагатор электронов проводимости и корреляционную функцию. Установлено свойство стационарности термодинамического потенциала по отношению к вариациям корреляционной функции.
\end{abstract}

Ключевые слова: сильно коррелированные электронные системы, уравнение Дайсона, функции Грина, примесная модель Андерсона.

\section{1. ВВЕДЕНИЕ}

Примесная модель Андерсона [1] была предложена в 1961 г. для описания сильно коррелированных электронных систем. Эта модель описывает систему свободных электронов проводимости, взаимодействующих с системой электронов, принадле-

* Институт прикладной физики АН Молдовы, Кишинев, Молдова

†Объединенный институт ядерных исследований, г. Дубна, Московская обл., Россия. E-mail: moskalen@thsun1.jinr.ru

${ }^{\ddagger}$ University of Duisburg-Essen, Duisburg, Germany

$\S$ Технический университет Молдовы, Кишинев, Молдова

${ }^{\top}$ Dipartimento di Fisica E. R. Caianiello, Universitá degli Studi di Salerno and CNISM, Baronissi, Italy 
жащих $d$ - или $f$-оболочке примесного атома. Примесные электроны сильно коррелированы из-за сильного кулоновского отталкивания. Происходит гибридизация состояний этих двух подсистем электронов. В частности, эта модель используется для описания тяжелых фермионов, где локальной примесной орбиталью является $f$-орбиталь [2].

Интерес к примесной модели Андерсона возрос в связи с появлением теории динамического среднего поля (ТДСП), в которой бесконечномерные решеточные модели проецируются на эффективные примесные модели в зависимом от времени поле, которое определяется условиями самосогласованности [3], [4] таким образом, чтобы локальная функция Грина для решетки была равна примесной функции Грина. Массовый оператор решеточной модели, зависящий только от частоты и не зависящий от импульса, используется посредством уравнения Дайсона, для того чтобы завершить итерационный процесс ТДСП. В исследованиях примесной модели Андерсона также использовались методы уравнений движения для запаздывающих и опережающих функций Грина, предложенные Боголюбовым и Тябликовым [5] и развитые в работах [6], [7].

В настоящей работе мы предлагаем диаграммную теорию для примесной модели Андерсона, которая является альтернативной методу уравнений движения [8], [9].

Первая попытка развить диаграммную теорию для данной системы была предпринята в работе [10], где использовались кумулянтное разложение для средних статистических произведений трансфер-операторов Хаббарда и алгебра этих операторов. Другие диаграммные подходы, рассматривающие гибридизацию состояний в качестве возмущения при точном учете одноузельных корреляций, рассматривались в работах [11]-[13].

В настоящей работе мы применяем термодинамическую теорию возмущений для мацубаровских [14], [15] функций Грина, рассматривая гибридизационное взаимодействие электронов проводимости и примесных электронов в качестве возмущения.

Гамильтониан модели имеет вид

$$
\begin{gathered}
H=H_{0}+H_{\mathrm{int}}, \quad H_{0}=H_{0}^{c}+H_{0}^{f}, \\
H_{0}^{c}=\sum_{\mathbf{k}, \sigma} \epsilon(\mathbf{k}) C_{\mathbf{k} \sigma}^{+} C_{\mathbf{k} \sigma}, \quad H_{0}^{f}=\epsilon_{f} \sum_{\sigma} f_{\sigma}^{+} f_{\sigma}+U n_{\uparrow}^{f} n_{\downarrow}^{f}, \\
H_{\mathrm{int}}=\frac{1}{\sqrt{N}} \sum_{\mathbf{k}, \sigma}\left(V_{\mathbf{k} \sigma} f_{\sigma}^{+} C_{\mathbf{k} \sigma}+V_{\mathbf{k} \sigma}^{*} C_{\mathbf{k} \sigma}^{+} f_{\sigma}\right), \quad n_{\sigma}^{f}=f_{\sigma}^{+} f_{\sigma},
\end{gathered}
$$

где $C_{\mathbf{k} \sigma}\left(C_{\mathbf{k} \sigma}^{+}\right)$и $f_{\sigma}\left(f_{\sigma}^{+}\right)$- соответственно операторы уничтожения (рождения) электронов проводимости и примесных электронов со спином $\sigma, \epsilon(\mathbf{k})$ - зонная энергия состояния $(\mathbf{k}, \sigma)$ электронов проводимости, $\epsilon_{f}-$ энергия локального уровня $f$-электронов примеси, $U$ - одноузельное отталкивание примесных электронов, $N$ число узлов кристаллической решетки. Обе энергии отсчитываются от химического потенциала $\mu$ системы. В качестве возмущения $H_{\text {int }}$ рассматривается взаимодействие гибридизации между электронами проводимости и примесными электронами. Кулоновское отталкивание между примесными электронами является слишком 
сильным, для того чтобы оно считалось возмущением, поэтому оно должно быть включено в основной гамильтониан $H_{0}$. Наличие этого члена делает невозможным использование обычной теоремы Вика для локальных электронов. Поэтому прежде всего мы формулируем обобщенную теорему Вика (ОТВ) для локальных электронов, сохраняя обычную теорему Вика для электронов проводимости. Сформулированная нами ОТВ фактически является тождеством, определяющим неприводимые функции Грина или кумулянты Кубо для электронов примеси.

Например, хронологическое среднее от четырех локальных операторов с матрицей плотности нулевого приближения имеет вид [16]

$$
\left\langle T f_{1} f_{2} \bar{f}_{3} \bar{f}_{4}\right\rangle_{0}=\left\langle T f_{1} \bar{f}_{4}\right\rangle_{0}\left\langle T f_{2} \bar{f}_{3}\right\rangle_{0}-\left\langle T f_{1} \bar{f}_{3}\right\rangle_{0}\left\langle T f_{2} \bar{f}_{4}\right\rangle_{0}+g_{2}^{(0) \mathrm{ir}}(1,2 \mid 3,4),
$$

где $\langle\cdot\rangle_{0}$ означает термодинамическое усреднение по нулевой матрице плотности. Первые два члена правой части равенства (2) являются вкладами обычного виковского типа, тогда как последний член, называемый нами неприводимой функцией Грина и являющийся кумулянтом Кубо, содержит спиновые, зарядовые и парные корреляции локализованных электронов. В частном случае, когда статистическое усреднение берется от произведения шести электронных $f$-операторов $\left\langle T f_{1} f_{2} f_{3} \bar{f}_{4} \bar{f}_{5} \bar{f}_{6}\right\rangle_{0}$, правая часть формулы вида (2) будет содержать 3 ! членов, каждый из которых есть произведение трех одночастичных функций Грина, девять членов в виде произведения одночастичной и двухчастичной неприводимых функций Грина и один член - неприводимую трехчастичную функцию Грина $g_{3}^{(0) \mathrm{ir}}(1,2,3$ | $4,5,6)$. Многочастичные неприводимые функции Грина $g_{n}^{(0) \mathrm{ir}}(1, \ldots, n \mid n+1, \ldots, 2 n)$, которые появляются в $n$-м порядке теории возмущения, определяют структуру новой диаграммной техники. Подобное определение неприводимых функций Грина мы использовали при обсуждении модели Хаббарда и других моделей сильно коррелированных электронов [17]-[21]. Гамильтониан $H_{0}$ локализованных электронов может быть диагонализован с помощью трансфер-операторов Хаббарда $\chi^{\mathrm{mn}}[22]$. Используя эти операторы, можно легко вычислить и простейшие неприводимые функции Грина.

\section{2. ДИАГРАММНАЯ ТЕОРИЯ}

В представлении взаимодействия полная мацубаровская одночастичная функция Грина имеет вид

$$
g_{\sigma \sigma^{\prime}}\left(\tau-\tau^{\prime}\right)=-\left\langle T f_{\sigma}(\tau) \bar{f}_{\sigma^{\prime}}\left(\tau^{\prime}\right) U(\beta)\right\rangle_{0}^{\mathrm{c}},
$$

где индекс "c" означает связность диаграмм, операторы берутся в представлении взаимодействия, $T$ - оператор хронологического упорядочения.

Введем локальный оператор для электронов проводимости

$$
b_{\sigma}=\frac{1}{\sqrt{N}} \sum_{\mathbf{k}} V_{\mathbf{k}} C_{\mathbf{k} \sigma}
$$

и соответствующую полную функцию Грина

$$
G_{\sigma \sigma^{\prime}}\left(\tau-\tau^{\prime}\right)=-\left\langle T b_{\sigma}(\tau) \bar{b}_{\sigma^{\prime}}\left(\tau^{\prime}\right) U(\beta)\right\rangle_{0}^{\mathrm{c}},
$$


где $U(\beta)$ - оператор эволюции:

$$
U(\beta)=T \exp \left[-\int_{0}^{\beta} H_{\mathrm{int}}(\tau) d \tau\right] .
$$

Поскольку матричный элемент гибридизации $V_{\mathbf{k}}$ связан с определением локального оператора (4), целесообразно ввести новый параметр $\lambda$, соответствующий каждой вершине диаграммы. Поэтому порядок теории возмущений будет определяться параметром $\lambda$, а не матричным элементом гибридизации $V_{\mathbf{k}}$, так как $V_{\mathbf{k}}$ может присутствовать даже в нулевом приближении для функции Грина. На последнем этапе вычислений параметр $\lambda$ будет полагаться равным единице.

В нулевом приближении теории возмущений функция Грина локальных электронов имеет вид $(\bar{\sigma}=-\sigma)$

$$
\begin{gathered}
g_{\sigma \sigma^{\prime}}^{0}(i \omega)=\delta_{\sigma \sigma^{\prime}} g_{\sigma}^{0}(i \omega), \quad g_{\sigma}^{0}(i \omega)=\frac{1-n_{\bar{\sigma}}}{i \omega-\epsilon_{f}}+\frac{n_{\bar{\sigma}}}{i \omega-\epsilon_{f}-U}, \\
n_{\bar{\sigma}}=\frac{e^{-\beta \epsilon_{f}}+e^{-\beta\left(2 \epsilon_{f}+U\right)}}{Z_{0}} \\
Z_{0}=1+2 e^{-\beta \epsilon_{f}}+e^{-\beta\left(2 \epsilon_{f}+U\right)}
\end{gathered}
$$

где $\omega \equiv \omega_{n}=(2 n+1) \pi / \beta$ - нечетные частоты Мацубары.

Для электронов проводимости имеем

$$
G_{\sigma \sigma^{\prime}}^{0}(i \omega)=\delta_{\sigma \sigma^{\prime}} G_{\sigma}^{0}(i \omega), \quad G_{\sigma}^{0}(i \omega)=\frac{1}{N} \sum_{\mathbf{k}} \frac{\left|V_{\mathbf{k}}\right|^{2}}{i \omega-\epsilon(\mathbf{k})} .
$$

Наличие в определении функции Грина $G_{\sigma}^{0}$ квадрата матричного элемента гибридизации есть следствие нашего определения (4), а не влияния возмущения.

На рис. 1 и 2 представлены результаты термодинамической теории возмущения для одночастичных функций Грина. Двойные сплошные и пунктирные линии изображают ренормированные, а тонкие линии - нулевые пропагаторы электронов проводимости и примеси. Линии соединяют крестики, которые показывают состояния примеси. Крестики снабжены двумя стрелками, одна из которых входящая, а другая выходящая, изображающими уничтожение и рождение электрона соответственно. Крестики являются вершинами диаграмм, и множитель $\lambda$ соответствует каждой вершине. Индекс $n$ обозначает $\left(\sigma_{n}, \tau_{n}\right)$ в диаграмме. Подразумевается суммирование по $\sigma_{n}$ и интегрирование по $\tau_{n}$. Прямоугольники с $2 n$ индексами и крестиками изображают неприводимые функции Грина $g_{n}^{(0) \text { ir }}(1, \ldots, n \mid n+1, \ldots, 2 n)$. Знак каждой диаграммы определяется четностью перестановок операторов Ферми, выполненных при построении диаграммы. Используя правила Фейнмана для построения диаграмм, согласно рис. 1 можно установить уравнение:

$$
\begin{aligned}
& G_{\sigma \sigma^{\prime}}\left(\tau-\tau^{\prime} \mid \lambda\right)=G_{\sigma \sigma^{\prime}}^{0}\left(\tau-\tau^{\prime}\right)+ \\
& \quad+\sum_{\sigma_{1}, \sigma_{2}} \int_{0}^{\beta} d \tau_{1} \int_{0}^{\beta} d \tau_{2} G_{\sigma \sigma_{1}}^{0}\left(\tau-\tau_{1}\right) \lambda g_{\sigma_{1} \sigma_{2}}\left(\tau_{1}-\tau_{2} \mid \lambda\right) \lambda G_{\sigma_{2} \sigma^{\prime}}^{0}\left(\tau_{2}-\tau^{\prime}\right) .
\end{aligned}
$$




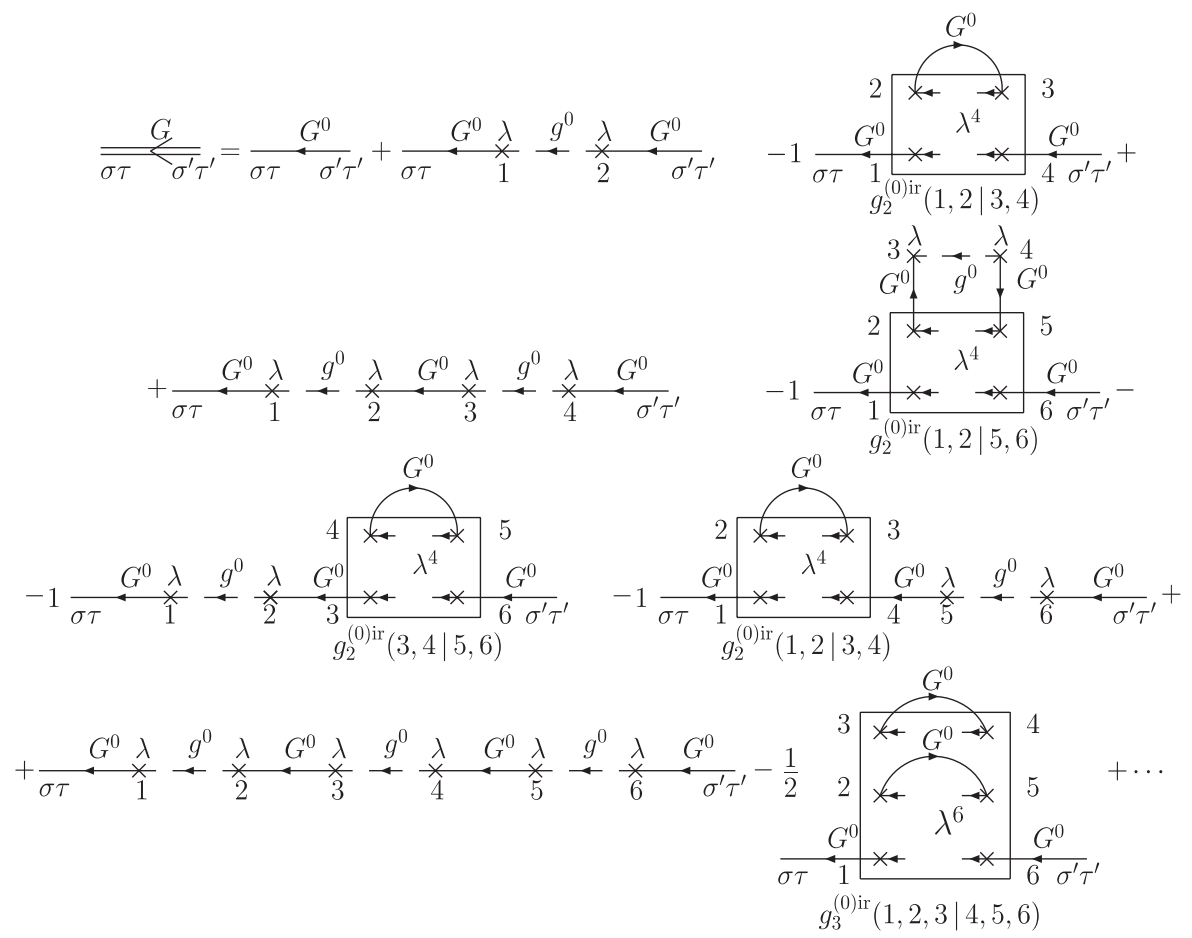

Рис. 1. Диаграммы первых порядков теории возмущений для пропагатора электронов проводимости. Тонкие сплошные линии изображают пропагаторы нулевого приближения для электронов проводимости, а пунктирные линии соответствующие пропагаторы для примесных электронов. Двойные линии изображают полные пропагаторы.

На основании диаграмм, приведенных на рис. 2, можно установить следующее уравнение типа уравнения Дайсона для $g_{\sigma \sigma^{\prime}}$ :

$$
\begin{aligned}
& g_{\sigma \sigma^{\prime}}\left(\tau-\tau^{\prime} \mid \lambda\right)=\Lambda_{\sigma \sigma^{\prime}}\left(\tau-\tau^{\prime} \mid \lambda\right)+ \\
& \quad+\sum_{\sigma_{1}, \sigma_{2}} \int_{0}^{\beta} d \tau_{1} \int_{0}^{\beta} d \tau_{2} \Lambda_{\sigma \sigma_{1}}\left(\tau-\tau_{1}\right) \lambda G_{\sigma_{1} \sigma_{2}}^{0}\left(\tau_{1}-\tau_{2}\right) \lambda g_{\sigma_{2} \sigma^{\prime}}\left(\tau_{2}-\tau^{\prime}\right),
\end{aligned}
$$

где

$$
\Lambda_{\sigma \sigma^{\prime}}\left(\tau-\tau^{\prime} \mid \lambda\right)=g_{\sigma \sigma^{\prime}}^{0}\left(\tau-\tau^{\prime}\right)+Z_{\sigma \sigma^{\prime}}\left(\tau-\tau^{\prime} \mid \lambda\right)
$$

$Z_{\sigma \sigma^{\prime}}$ - новая корреляционная функция, которая представляет собой бесконечную сумму сильносвязных неприводимых функций Грина. Как уже подчеркивалось выше, эта функция содержит все спиновые, зарядовые и парные флуктуации и является основным элементом нашей диаграммной техники.

На рис. 3 приведено диаграммное представление корреляционной функции $\Lambda_{\sigma \sigma^{\prime}}\left(\tau-\tau^{\prime} \mid \lambda\right)$. 


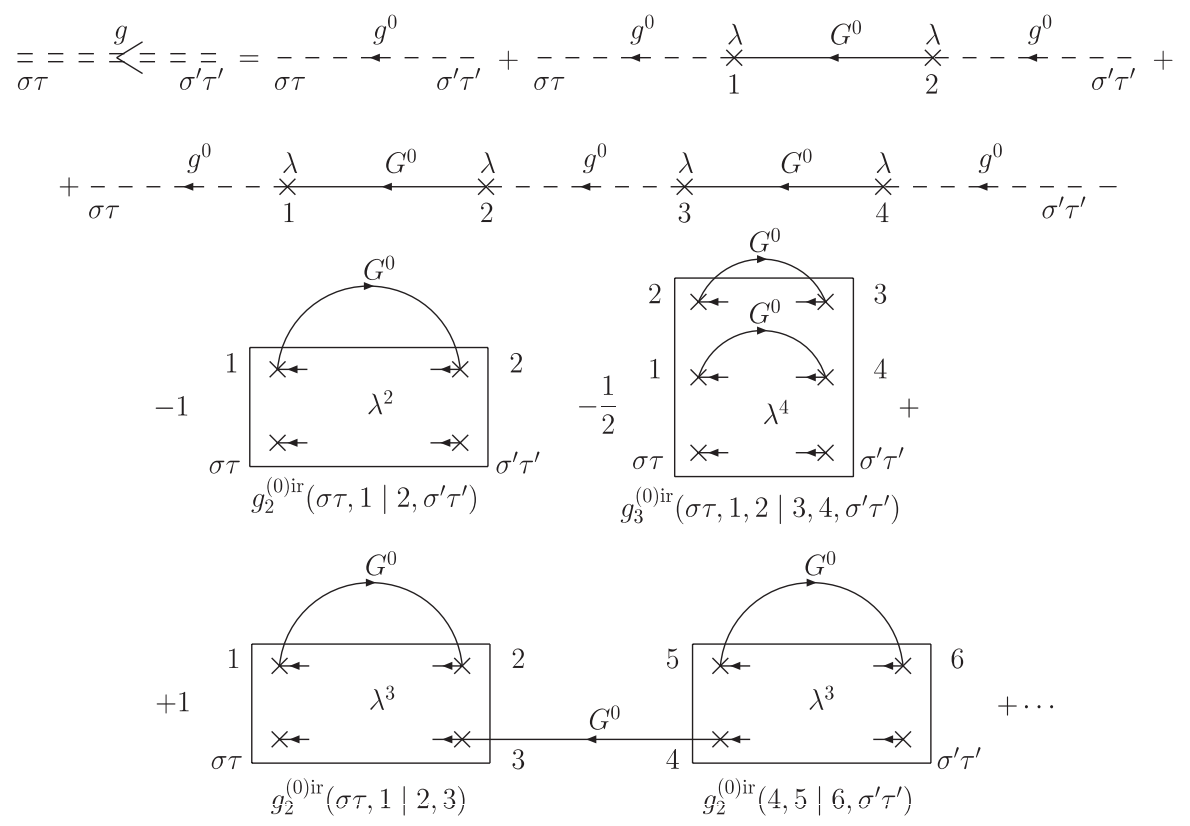

Рис. 2. Диаграммы для примесного электронного пропагатора $g_{\sigma \sigma^{\prime}}\left(\tau-\tau^{\prime}\right)$. Последние три диаграммы содержат корреляционные вклады. Две из них являются сильносвязными, а последняя является слабосвязной.
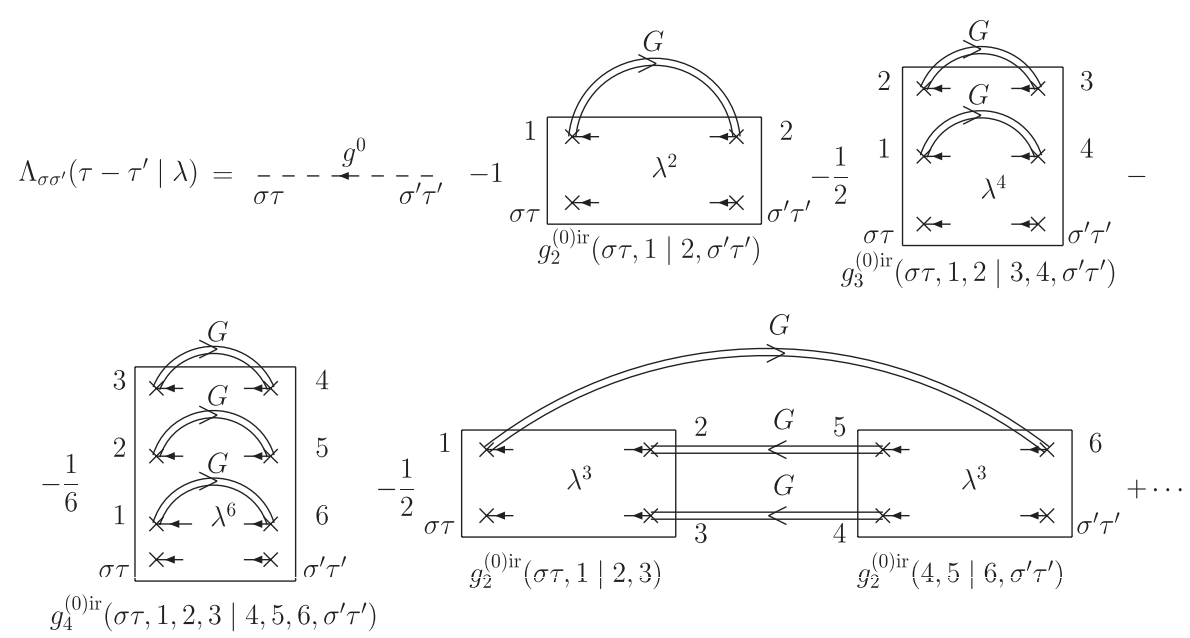

Рис. 3. Диаграммы, представляющие корреляционную функцию $\Lambda_{\sigma \sigma^{\prime}}$. Двойные сплошные линии изображают полные функции Грина электронов проводимости $G_{\sigma \sigma^{\prime}}\left(\tau-\tau^{\prime} \mid \lambda\right)$. Предполагается, что аргументы неприводимых функций Грина расположены по часовой стрелке. 
Разложение в ряд полного пропагатора $G_{\sigma \sigma^{\prime}}\left(\tau-\tau^{\prime}\right)$ дает наиболее детальное представление об этой величине.

Используя представление Фурье для мацубаровских функций Грина, с учетом уравнений (9), (10) получаем

$$
\begin{aligned}
G_{\sigma}(i \omega \mid \lambda) & =\frac{G_{\sigma}^{0}(i \omega)}{1-\Lambda_{\sigma}(i \omega \mid \lambda) G_{\sigma}^{0}(i \omega) \lambda^{2}} \\
g_{\sigma}(i \omega \mid \lambda) & =\frac{\Lambda_{\sigma}(i \omega \mid \lambda)}{1-\Lambda_{\sigma}(i \omega \mid \lambda) G_{\sigma}^{0}(i \omega) \lambda^{2}}
\end{aligned}
$$

Уравнение (12), полученное для пропагатора электронов проводимости, является уравнением Дайсона с массовым оператором, который определяется корреляционной функцией примесных электронов

$$
\Sigma_{\sigma}(i \omega \mid \lambda)=\lambda^{2} \Lambda_{\sigma}(i \omega \mid \lambda)
$$

В случае, если параметр $\lambda$ равен единице, величины $\Sigma_{\sigma}$ и $\Lambda_{\sigma}$ совпадают.

Уравнение (13) для примесных электронов является уравнением типа уравнения Дайсона. При $\lambda=1$ оно совпадает с другими подобными уравнениями, полученными для других сильно коррелированных моделей [17]-[21]. В уравнениях (12) и (13) параметр $\lambda$ может быть положен равным единице.

\section{3. ТЕРМОДИНАМИЧЕСКИЙ ПОТЕНЦИАЛ}

Термодинамический потенциал нашей сильно коррелированной системы равен

$$
\begin{aligned}
F & =F_{0}-\frac{1}{\beta} \ln \langle U(\beta)\rangle_{0}, \\
F_{0} & =-\frac{1}{\beta} \ln Z_{0}-\frac{2}{\beta} \sum_{\mathbf{k}} \ln \left[1+e^{-\beta \epsilon(\mathbf{k})}\right] .
\end{aligned}
$$

Диаграммы, которые определяют среднее значение оператора эволюции $\langle U(\beta)\rangle_{0}$, лишены внешних линий и называются вакуумными. Среди этих диаграмм имеются связные и несвязные диаграммы. Несвязные диаграммы могут быть просуммированы, в результате чего возникает экспонента от суммы связных диаграмм. Результат такого суммирования может быть сформулирован в виде теоремы о связных диаграммах:

$$
\langle U(\beta)\rangle_{0}=e^{\langle U(\beta)\rangle_{0}^{c}}
$$

где $\langle U(\beta)\rangle_{0}^{\text {c }}$ - бесконечная сумма связных вакуумных диаграмм. Эта величина равна нулю, если гибридизация отсутствует. 


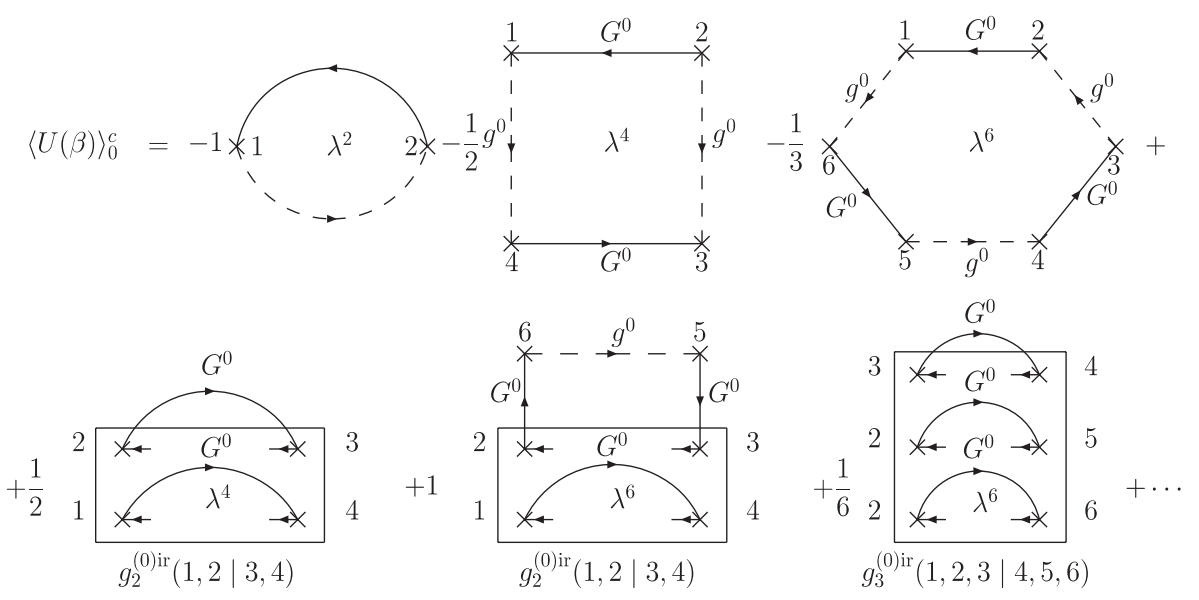

Рис. 4. Связные вакуумные диаграммы второго, четвертого и шестого порядков теории возмущений.

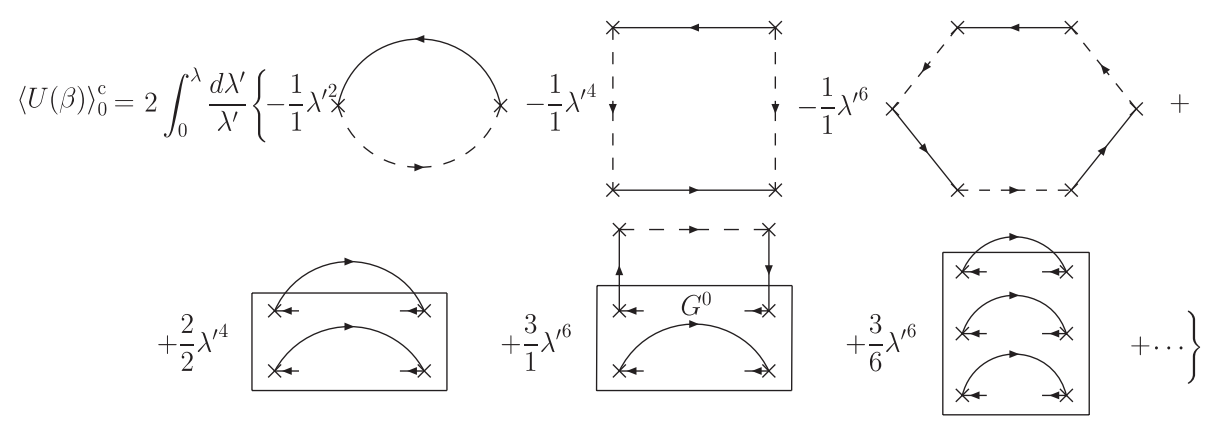

Рис. 5. Результат интегрирования вакуумных диаграмм по константе $\lambda=1$.

В силу (16) имеем

$$
F=F_{0}-\frac{1}{\beta}\langle U(\beta)\rangle_{0}^{\mathrm{c}} .
$$

На рис. 4 представлены простейшие вакуумные диаграммы. Первые три диаграммы являются диаграммами цепочного типа, они получены от тех вкладов, которые определяются обычной теоремой Вика. Однако последние три диаграммы содержат корреляционные функции и происходят от дополнительных членов ОТВ.

В вакуумных диаграммах присутствует множитель $1 / n$, где $n$ - порядок теории возмущений. Этот множитель препятствует процессу суммирования диаграмм и может быть устранен при помощи известного приема интегрирования по введенной нами константе связи $\lambda$. Результат этого интегрирования приведен на рис. 5. После этого преобразования мы можем воспользоваться диаграммным определением полного пропагатора $G_{\sigma}$ (см. рис. 1$)$ и корреляционной функции $\Lambda_{\sigma}$ (см. рис. 3 ) и комбинировать их вклады таким образом, чтобы получить диаграммы рис. 5. 
Можно убедиться в том, что любая диаграмма, приведенная на рис. 5 и имеющая порядок $n$ теории возмущений, может быть получена как произведение вклада порядка $n_{1}$ из диаграммы рис. 1 и вклада порядка $n_{2}$ из диаграммы рис. 3 при условии, что $n_{1}+n_{2}+2=n$. Это совпадение имеет место при условии, что учитываются все возможности такого построения вакуумных диаграмм при различных $n_{1}$ и $n_{2}$. В результате мы получаем

$$
\begin{aligned}
\langle U(\beta)\rangle_{0}^{\mathrm{c}} & =-2 \sum_{\sigma_{1}, \sigma_{2}} \int_{0}^{\beta} d \tau_{1} \int_{0}^{\beta} d \tau_{2} \int_{0}^{\lambda} \frac{d \lambda^{\prime}}{\lambda^{\prime}} G_{\sigma_{1} \sigma_{2}}\left(\tau_{1}-\tau_{2} \mid \lambda^{\prime}\right) \Sigma_{\sigma_{2} \sigma_{1}}\left(\tau_{2}-\tau_{1} \mid \lambda^{\prime}\right)= \\
& =-2 \sum_{\sigma} \sum_{\omega} \int_{0}^{\lambda} \frac{d \lambda^{\prime}}{\lambda^{\prime}} G_{\sigma}\left(i \omega \mid \lambda^{\prime}\right) \Sigma_{\sigma}\left(i \omega \mid \lambda^{\prime}\right) .
\end{aligned}
$$

Термодинамический потенциал принимает вид

$$
F=F_{0}+\frac{2}{\beta} \sum_{\sigma} \sum_{\omega} \int_{0}^{\lambda} \frac{d \lambda^{\prime}}{\lambda^{\prime}} G_{\sigma}\left(i \omega \mid \lambda^{\prime}\right) \Sigma_{\sigma}\left(i \omega \mid \lambda^{\prime}\right)
$$

Отсюда следует

$$
\lambda \frac{\partial F}{\partial \lambda}=\frac{2}{\beta} \sum_{\sigma} \sum_{\omega} G_{\sigma}(i \omega \mid \lambda) \Sigma_{\sigma}(i \omega \mid \lambda)
$$

Выражение (19) для термодинамического потенциала содержит дополнительное интегрирование по константе связи $\lambda$ и поэтому оказывается более громоздким.

Как было показано в работе [23] для некоррелированных многоэлектронных систем потенциал (19) можно записать в более удобной форме. Для этого рассмотрим выражение

$$
Y=-\frac{1}{\beta} \sum_{\sigma} \sum_{\omega} e^{i \omega 0^{+}}\left\{\ln \left[G_{\sigma}^{0}(i \omega) \Sigma_{\sigma}(i \omega \mid \lambda)-1\right]+G_{\sigma}(i \omega \mid \lambda) \Sigma_{\sigma}(i \omega \mid \lambda)\right\}+Y^{\prime},
$$

которое представляет собой обобщение для сильно коррелированных систем выражения, предложенного Латтинжером и Уордом. В (21) $Y^{\prime}$ - сумма скелетных сильносвязных диаграмм (см. рис. 6), двойным линиям которых соответствуют полная одночастичная функция Грина $G_{\sigma}$ электронов проводимости.

На рис. 6 представлены некоторые простейшие диаграммы. Эти диаграммы зависят от константы взаимодействия $\lambda$ не только через множители перед диаграммами, но также и через полные пропагаторы $G_{\sigma}(i \omega \mid \lambda)$.

В силу (12), (14) и (21) получаем

$$
\frac{\partial Y}{\partial \Sigma_{\sigma}(i \omega \mid \lambda)}=-\frac{1}{\beta} \Sigma_{\sigma}(i \omega \mid \lambda) G_{\sigma}^{2}(i \omega \mid \lambda)+\frac{\partial Y^{\prime}}{\partial \Sigma_{\sigma}(i \omega \mid \lambda)}
$$



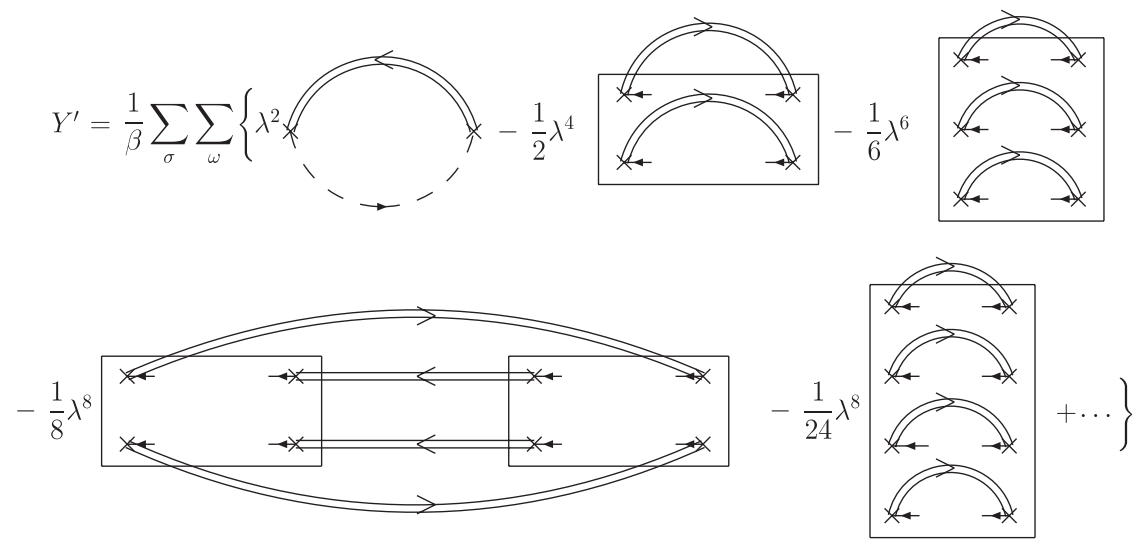

Рис. 6. Замкнутые связные скелетные диаграммы. Двойные сплошные линии соответствуют полным пропагаторам $G_{\sigma}(i \omega \mid \lambda)$ электронов проводимости. Прямоугольники соответствуют корреляционным функциям скоррелированных электронов.

Из (22) согласно рис. 3, рис. 6 и определению (14) следует равенство

$$
\frac{\partial Y^{\prime}}{\partial G_{\sigma}(i \omega \mid \lambda)}=\frac{\lambda^{2}}{\beta} \Lambda_{\sigma}(i \omega \mid \lambda)=\frac{\Sigma_{\sigma}(i \omega \mid \lambda)}{\beta} .
$$

Как следствие получаем свойство стационарности функционала $Y$ относительно изменения массового оператора

$$
\frac{\partial Y}{\partial \Sigma_{\sigma}(i \omega \mid \lambda)}=0
$$

Найдем теперь производную $\partial Y / \partial \lambda$. Используя свойство стационарности функционала $Y$, мы можем пренебречь зависимостью $\Sigma_{\sigma}$ и $G_{\sigma}$ от $\lambda$ и тем самым принять во внимание только явную зависимость от $\lambda$ в функционале $Y^{\prime}$, представленном на рис. 6. С помощью этого рисунка легко получить:

$$
\left.\lambda \frac{\partial Y}{\partial \lambda}\right|_{\Sigma}=\left.\lambda \frac{\partial Y^{\prime}}{\partial \lambda}\right|_{\Sigma}=\frac{2}{\beta} \sum_{\sigma} \sum_{\omega} G_{\sigma}(i \omega \mid \lambda) \Sigma_{\sigma}(i \omega \mid \lambda) .
$$

Из (20) и (25) следует

$$
\lambda \frac{\partial F}{\partial \lambda}=\lambda \frac{\partial Y}{\partial \lambda}
$$

откуда вытекает равенство

$$
F(\lambda)=Y(\lambda)+\text { const }
$$


При $\lambda=0$ имеем $Y(0)=0$ и $F(0)=F_{0}$. Следовательно, const $=F_{0}$. Окончательно имеем

$$
F=F_{0}+Y
$$

\section{4. ЗАКЛЮЧЕНИЕ}

В настоящей работе был вычислен термодинамический потенциал сильно коррелированной системы, описанной примесной моделью Андерсона. Нами была сформулирована новая диаграммная техника для сильно коррелированных фермионов и определены корреляционная функция локализованных электронов и массовый оператор электронов проводимости. Эти последние величины совпадают. Сформулированы уравнения типа уравнения Дайсона для пропагаторов обеих подсистем. На основании этой диаграммной техники мы впервые установили точное выражение термодинамического потенциала через полный пропагатор $G_{\sigma}$ и массовый оператор $\Sigma_{\sigma}$ электронов проводимости и затем доказали свойство стационарности термодинамического потенциала относительно вариации массового оператора. Этот результат является обобщением соответствующей теоремы Латтинжера и Уорда [23], установленной для не сильно коррелированных систем. Обобщенная теорема может оказаться полезной при исследовании термодинамических свойств, например теплоемкости, сильно коррелированных систем.

Благодарности. Авторы выражают глубокую благодарность профессору Н. М. Плакиде за обсуждение работы и ценные замечания. Работа В. А. Москаленко и П. Ентель была поддержана Программой "Гейзенберг-Ландау". В. А. Москаленко благодарит отдел теоретической физики университета Duisburg-Essen за гостеприимство и финансовую поддержку.

\section{Список литературы}

[1] P. W. Anderson, Phys. Rev., 124:1 (1961), 41-53.

[2] A. C. Hewson, The Kondo Problem to Heavy Fermions, Cambridge Studies in Magnetism, Cambridge Univ. Press, Cambridge, 1993.

[3] A. Georges, G. Kotliar, W. Krauth, M. J. Rozenberg, Rev. Modern Phys., 68:1 (1996), $13-125$.

[4] G. Kotliar, D. Vollhardt, Phys. Today, 57:3 (2004), 53-59.

[5] Н. Н. Боголюбов, С. В. Тябликов, ДАН СССР, 126 (1959), 53-56.

[6] Д. Н. Зубарев, УФН, 71:2 (1960), 71-116.

[7] В. Л. Бонч-Бруевич, С. В. Тябликов, Метод функиий Грина в статистической механике, Физматлит, М., 1961.

[8] K. G. Wilson, Rev. Modern Phys., 47:4 (1975), 773-840.

[9] T. A. Costi, A. C. Hewson, V. Zlatic, J. Phys., 6:13 (1994), 2519-2558.

[10] А. Ф. Барабанов, К. А. Кикоин, Л. А. Максимов, ТМФ, 20:3 (1974), 364-380.

[11] H. Schoeller, G. Schön, Phys. Rev. B, 50:24 (1994), 18436-18452.

[12] J. König, J. Schmid, H. Schoeller, G. Schön, Phys. Rev. B, 54:23 (1996), 16820-16837.

[13] N. Sivan, N. S. Wingreen, Phys. Rev. B, 54:16 (1996), 11622-11629.

[14] T. Matsubara, Progr. Theoret. Phys., 14:4 (1955), 351-378.

[15] А. А. Абрикосов, Л. П. Горьков, И. Е. Дзялошинский, Методы квантовой теории поля в статистической физике, Добросвет, М., 1998. 
[16] В. А. Москаленко, П. Ентель, Д.Ф. Дигор, Л. А. Дохотару, Р. Читро, ТМФ, 155:3 (2008), 474-497.

[17] М. И. Владимир, В. А. Москаленко, ТМФ, 82:3 (1990), 428-437.

[18] С. И. Вакару, М. И. Владимир, В. А. Москаленко, ТМФ, 85:2 (1990), 248-257.

[19] Н. Н. Боголюбов, В. А. Москаленко, ТМФ, 86:1 (1991), 16-30.

[20] Н. Н. Боголюбов, В. А. Москаленко, ТМФ, 92:2 (1992), 182-190.

[21] V. A. Moskalenko, P. Entel, D. F. Digor, Phys. Rev. B, 59:1 (1999), 619-635.

[22] J. Hubbard, Proc. Roy. Soc. Ser. A, 276:1365 (1963), 238-257; 281:1386 (1964), 401-419; 285:1403 (1965), 542-560.

[23] J. M. Luttinger, J. C. Ward, Phys. Rev. B, 118:5 (1960), 1417-1427.

Поступила в редакцию 2.08.2008 